cluded and no detailed discussion of the somewhat analogous exponentially decaying solutions introduced by Gamow for radioactive nuclei seems to be available. The resonance discussions of Morse and Stueckelberg and London's considerations on the method of perturbed stationary state functions are, on the other hand, considered in some detail and should prove of value to chem. ists. The discussion of nuclear scattering at the end of the book shows the nature of the mathematical methods used but it not sufficiently detailed to indicate whether the theory is applicable to the nucleus. The authors content themselves with brief statements about very good agreement with experiment. Although it is certain that for distant nuclear collisions wave mechanics works, too much optimism about its universal applicability can be scarcely justified at present. Thus Taylor's results indicate a systematic variation of nuclear radius of helium with velocity, and the interpretation of the results seems to be largely a matter of faith.

\title{
G. BREIT
}

Höhere Algebra. By Helmut Hasse. Volume I, Lineare Gleichungen. Sammlung Göschen. Berlin and Leipzig, de Gruyter, 1933. 152 pp.

This is a second edition of an excellent book first published in 1926 and reviewed in this Bulletin, vol. 33 (1927), p. 251. It contains an introduction to the study of algebraic systems; fields, domains of integrity, and groups. The principal part of the book is devoted to the solution of systems of linear equations. The author in Chapter 3 departs from the usual presentation in first developing the theory without the use of determinants by means of the notion of equivalence of systems and the Toeplitz procedure of reduction. Chapter 4 contains the usual treatment in terms of determinants. The changes from the first edition consist chiefly in the correction of typographical errors.

\section{H. T. ENGSTROM}

Minimum Decompositions into Fifth Powers. By L. E. Dickson. Mathematical Tables, British Association for the Advancement of Science, vol. 3. London, 1933.

A great number of numerical investigations have been carried out in connection with Waring's problem. There is the table of Dase (1851) on the representation of numbers as a sum of cubes for all integers up to 12000 and von Sterneck's extension (1903) to 40000 . For biquadratic representations Bretschneider (1853) gave a table to 4100 , and for fifth powers there is Wieferich's table to 3011 .

Dickson's table extends to the imposing number 300000 . Through various new and ingenious short-cuts it has been possible to reduce the computations and also the tabulation of the results. The table is constructed to supplement the analytic theory of Waring's problem and to verify a hypothesis of Dickson, that the integers for which a maximal number of components is required are relatively small. Those interested in the present state of the problem can find an excellent account in Dickson's review: Recent progress on Waring's theorem and its generalizations (this Bulletin, 1933, pp. 701-727). 